\title{
Mitral valve repair provides improved outcome over replacement in active infective endocarditis
}

\author{
Elfriede Ruttmann, MD, ${ }^{a}$ Christina Legit, MD, ${ }^{a}$ Gerhard Poelzl, MD, ${ }^{b}$ Silvana Mueller, MD, ${ }^{b}$ Orest Chevtchik, MD, ${ }^{a}$ \\ Marco Cottogni, MD, ${ }^{a}$, Hanno Ulmer, PhD, ${ }^{c}$ Othmar Pachinger, MD, ${ }^{b}$ Guenther Laufer, MD, and Ludwig C. Mueller, MD ${ }^{a}$
}

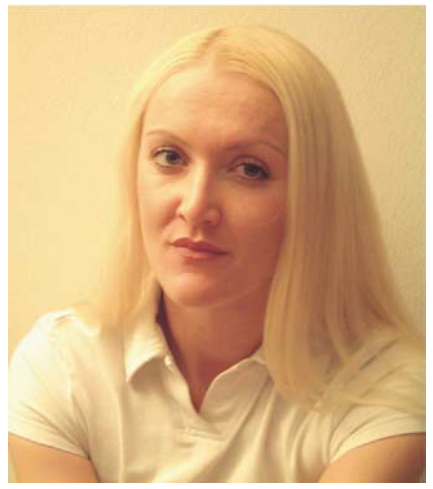

Dr Ruttmann
Objectives: Mitral repair in active infective endocarditis still remains controversial. Several studies demonstrate the feasibility of mitral repair in infective endocarditis; however, superiority of repair has never been shown. The aim of the investigation was to compare valve repair and valve replacement in respect to the extent of destruction and to analyze survival, recurrent endocarditis, and reoperation (event-free survival).

Methods: Sixty-eight consecutive patients underwent surgical intervention for mitral endocarditis. Thirty-four (50\%) patients had valve repair, and 34 (50\%) patients had valve replacement. Leaflet destruction involving at least one mitral leaflet was present in $15(44.1 \%)$ patients of the repair group and $11(32.4 \%)$ patients of the replacement group. Repair of the mitral annulus with pericardium was performed in $4(11.8 \%)$ patients in the repair group and $3(8.8 \%)$ patients in the replacement group. Patients in both groups were similar concerning the progression of valvular destructions and comorbidities.

Results: Hospital mortality was $11.8 \%$ (8 patients). No significant differences were found in all baseline parameters, with the exception of a higher incidence of previous septic embolism and sepsis in the repair group. Actuarial event-free survival at 1 year was $88.2 \%$ in the repair group compared with $67.7 \%$ in the replacement group, and 5-year event-free survival was $80.4 \%$ in the repair group and $54.6 \%$ in the replacement group $(P=.015)$. Mitral valve repair remained the superior treatment regarding event-free survival in the multivariate analysis (hazard ratio, $0.33 ; 95 \%$ confidence interval, $0.12-0.93 ; P=.02$ ).

Conclusions: Mitral valve repair offers excellent early and late results and is the preferable treatment option in the surgical therapy of native infective endocarditis.

From the Departments of Cardiac Surgery, ${ }^{\mathrm{a}}$ Cardiology, ${ }^{\mathrm{b}}$ and Medical Statistics, Informatics, and Health Economics, ${ }^{\mathrm{c}}$ Innsbruck Medical University, Innsbruck, Austria.

Received for publication Dec 30, 2004; revisions received Feb 22, 2005; accepted for publication March 10, 2005.

Address for reprints: Elfriede Ruttmann, MD, Department of Cardiac Surgery, Innsbruck Medical University, Anichstrasse 35, 6020 Innsbruck, Austria (E-mail: elfriede. ruttmann@uibk.ac.at).

J Thorac Cardiovasc Surg 2005;130:765-71

$0022-5223 / \$ 30.00$

Copyright () 2005 by The American Association for Thoracic Surgery

doi:10.1016/j.jtcvs.2005.03.016
$\mathrm{E}$ ven though there have been great improvements in general health care and antibiotic therapy, the incidence of infective endocarditis (IE) has not changed during the past decades. ${ }^{1}$

However, there has been a shift in the clinical appearance of IE. Underlying rheumatic valvular disease that was frequently observed in patients with IE during the 1980s has become rare in industrialized countries. ${ }^{2}$ As a predisposing factor, it has been replaced by other causes, such as intravenous drug abuse, immunosuppression, degenerative valvular disease, intravascular prostheses and devices, hemodialysis shunt infections, and other nosocomial infections. ${ }^{3}$

Mitral valve repair is a well-established treatment for noninfected valves and offers certain advantages. Because of preservation of the subvalvular apparatus and the resulting restoration of left ventricular function, mitral valve repair is favorable in the treatment of mitral regurgitation.

In mitral surgery for IE, prosthetic valve replacement has been the standard therapy for many years. Because of the decreasing incidence of underlying rheumatic disease and the convincing long-term results of repair of noninfected valves, ${ }^{4}$ mitral valve repair in patients with IE is still controversial. 
TABLE 1. Patient characteristics

\begin{tabular}{|c|c|c|c|}
\hline & $\begin{array}{l}\text { Mitral valve repair } \\
\qquad(\mathrm{n}=34)\end{array}$ & $\begin{array}{l}\text { Mitral valve replacement } \\
\qquad(\mathrm{n}=34)\end{array}$ & $P$ value \\
\hline Age, y & $51.5 \pm 17.0^{*}$ & $53.2 \pm 13.1$ & .840 \\
\hline Male sex & $22(64.7 \%)$ & $17(50.0 \%)$ & .383 \\
\hline Obesity, BMI $\geq 30$ kg/m²) & $3(8.8 \%)$ & $4(11.8 \%)$ & .721 \\
\hline Diabetes & $6(17.6 \%)$ & $6(17.6 \%)$ & .954 \\
\hline Chronic obstructive pulmonary disease & $6(17.6 \%)$ & $5(14.7 \%)$ & .701 \\
\hline Impaired renal function (creatinine $>2 \mathrm{mg} / \mathrm{dL}$ ) & $10(29.4 \%)$ & $12(35.3 \%)$ & .479 \\
\hline Preoperative kidney failure & $6(17.6 \%)$ & $3(8.8 \%)$ & .476 \\
\hline Ejection fraction & $49 \% \pm 12 \%$ & $53 \% \pm 12 \%$ & .197 \\
\hline NYHA stage (mean) & $2.80 \pm 0.87$ & $2.76 \pm 0.55$ & .968 \\
\hline NYHA stage IV (\%) & $7(20.6 \%)$ & $2(5.9 \%)$ & .121 \\
\hline Previous septic embolization & $15(44.2 \%)$ & $6(17.6 \%)$ & .027 \\
\hline Preexisting degenerative valvular disease & $15(44.2 \%)$ & $12(35.3 \%)$ & .840 \\
\hline EuroSCORE (mean) & $9.8 \pm 4.2$ & $9.7 \pm 3.8$ & .760 \\
\hline
\end{tabular}

BMI, Body mass index; NYHA, New York Heart Association. *Means \pm SD.

Dreyfus and colleagues ${ }^{5}$ were the first to demonstrate that valve repair is feasible in aortic and mitral endocarditis in 1990.

Several outcome studies $^{6-8}$ have confirmed that mitral valve repair is effective in patients with IE but were lacking comparable matched cohorts of patients undergoing mitral valve replacement for the same indication. ${ }^{6,7}$ Additionally, in most studies, patients with healed endocarditis and patients with underlying rheumatic disease were investigated and had limited sample sizes. ${ }^{9-11}$

Superiority of valve repair as a possible treatment option for active mitral endocarditis has never been confirmed.

At the Department of Cardiac Surgery at the University Hospital Innsbruck, a large number of patients with active mitral endocarditis have undergone operations during recent years. Approximately half of the patients underwent mitral repair.

The aims of our study were (1) to investigate whether there are differences in the extent of infectious destruction of mitral valves undergoing either repair or replacement and (2) to evaluate whether valve repair offers a benefit over replacement in acute IE in respect to perioperative mortality and long-term event-free survival.

\section{Methods}

From 1992 through 2004, a consecutive series of 74 patients underwent cardiac operations for active IE of the native mitral valve at the Cardiac Surgery Department of the University Hospital Innsbruck. Six patients with an underlying rheumatic valve stenosis were excluded from further analysis to avoid selection bias because of limited chance for sufficient valve repair.

A total of 68 patients (39 [57.4\%] male and 29 [42.6\%] female patients) were divided into 2 cohorts with regard to the type of mitral valve procedure performed: $34(50 \%)$ patients underwent mitral valve repair, and the remaining 34 patients underwent mitral valve replacement.
All patients were receiving antibiotic treatment and had to undergo an operation because of the failure of conservative therapy (persisting sepsis, embolic events, and hemodynamic instability). All patients described in our series were given diagnoses not only on the basis of the clinical Dukes criteria but also on the basis of preoperative and intraoperative echocardiography. ${ }^{12,13}$ Additionally, intraoperative bacterial cultures and histology of the excised valve tissue confirmed the diagnosis of active valvular infection.

All surgical procedures, both repair and replacement, were performed by experienced surgeons in the position of a consultant. From a technical aspect, radical debridement of the infective material without consideration of the resulting lesion and, in a second step, restoration of a morphologic and functional valve were performed.

Event-free survival was defined as the primary outcome measure to compare efficacy. Deaths of all causes, valvular reoperation, and recurrent endocarditis were regarded as events. KaplanMeier analysis, together with log-rank testing, was used to compare event-free survival between the 2 treatment options (repair vs replacement). The Pearson $\chi^{2}$ test or the Fisher exact test (when appropriate) was used to test for baseline differences in categoric variables. Continuous variables were tested with the Student $t$ test or the Mann-Whitney $U$ test (if assumption of Gaussian distribution was not fulfilled). The Cox proportional hazard model was used to assess the effect of treatment independently from other potential life-threatening predictors. Adjusted hazard ratios (HRs) and their $95 \%$ confidence intervals (CIs) were calculated. All variables that showed a significant effect on eventfree survival in the univariate Kaplan-Meier analysis were included in the multivariate Cox model.

Data documentation and statistical analysis were performed with SPSS software version 11.0 (SPSS Inc, Chicago, Ill).

\section{Results \\ Patient Characteristics}

The mean age of the patients was $51.5 \pm 17.0$ years in the repair group and $53.2 \pm 13.1$ years in the replacement group 
$(P=.840)$. The median time from onset of intravenous antibiotic treatment to surgical intervention was 11 days (0-44 days) in the repair group and 12 days (1-48 days) in the replacement group $(P=.31)$. The median time from definitive diagnosis (day of transesophageal echocardiography) to surgical intervention was 4 days (1-25 days) in the replacement group and 3.5 days (0-21 days) in the repair group $(P=.32)$.

A detailed preoperative patient description is displayed in Table 1.

Median follow-up was 37.7 months in the repair group and 44.5 months in the replacement group $(P=.240)$. Follow-up was performed by the referring cardiologists, including frequent echocardiography, and was $100 \%$ complete.

Renal failure was present in $6(17.6 \%)$ patients in the repair group and $3(8.8 \%)$ patients in the replacement group $(P=.476)$. Mean ejection fraction at the time of the operation was $49 \% \pm 12 \%$ in the repair group and $53 \% \pm$ $12 \%$ in the replacement group $(P=.197)$.

Mean cardiopulmonary bypass time was $166 \pm 63.2$ minutes in the repair group and $144.3 \pm 91.0$ minutes in the replacement group $(P=.260)$. Aortic crossclamping time was significantly longer in the repair group $(114.3 \pm 52.3$ vs $85.5 \pm 40.5$ minutes, $P=.014$ ).

Underlying degenerative mitral valve disease was present in $15(44.2 \%)$ patients in the repair group and 12 (35.3\%) patients in the replacement group $(P=.820)$.

In the mitral repair group, however, a higher number of patients had experienced preoperative septic embolism (cerebral and peripheral) than in the replacement group (44.2\% vs $17.6 \%, P=.027)$. Additionally, sepsis as the main indication for surgical intervention was significantly more common in the mitral repair group (50\% vs $26.1 \%, P=$ .018). Otherwise, the 2 treatment groups did not differ significantly concerning preoperative comorbidity, surgical risk (assessed by the EuroSCORE), or the extent of destruction of the mitral valve (Table 2).

Additional procedures, such as coronary artery bypass grafting, aortic valve replacement, and/or tricuspid valve repair (caused by double-valve endocarditis), were necessary in $13(38.2 \%)$ patients in the repair group and 13 (38.2\%) patients in the replacement group $(P=1.0)$.

At the time of the operation, valves were still found to be contaminated by infective organisms in $15(44.1 \%)$ patients in the repair group and $19(55.9 \%)$ patients in the replacement group $(P=.332)$. Staphylococcus species were isolated in blood, valve cultures, or both in $19(55.9 \%)$ patients in the repair group and $13(38.2 \%)$ patients in the replacement group $(P=.145)$. Leaflet destruction was present in $15(44.1 \%)$ patients in the repair group and 11 (32.4\%) patients in the replacement group $(P=.394)$. Perivalvular destruction and abscesses requiring annular reconstruction with autologous glutaraldehyde-fixed pericardium were per- formed in $4(11.8 \%)$ patients in the repair group and 3 $(8.8 \%)$ patients in the replacement group $(P=.659)$. Commissural affection was present in $3(8.8 \%)$ patients in the repair group and $4(11.7 \%)$ patients in the replacement group $(P=.73)$.

Mitral ring annuloplasty was performed in 19 (55.9\%) patients (Carpentier Edwards in 13 patients, Sequin in 5 patients, and St Jude Tailor in 1 patient) in the repair group. Ring annuloplasty was indicated when sufficient coaptation of leaflets was not achievable without it and was frequently necessary in patients showing annular dilatation. Transposition of secondary chordae in the primary position was performed in 4 patients, and artificial chordae were used in 1 male patient in the repair group.

In the replacement group $28(82.4 \%)$ patients received a mechanical mitral valve prosthesis, and the remaining 6 (17.6\%) patients underwent biologic mitral valve replacement.

\section{Perioperative Mortality}

Overall perioperative mortality was $11.8 \%$ (8 patients, 4 patients in the repair group and 4 patients in the replacement group; $P=1.0$ ).

One female patient had double-valve endocarditis (aortic and mitral endocarditis, methicillin-resistant Staphylococcus aureus, and Aspergillus fumigatus) and invasive pulmonary aspergillosis after she had undergone her third kidney transplantation. She underwent aortic root replacement with a porcine xenograft root (Freestyle; Medtronic Minneapolis, Minn) because of destructive double-valve endocarditis and a complex mitral valve repair. She died on the fifth postoperative day of invasive aspergillosis. Another female patient was given a diagnosis of acute mitral valve endocarditis 1 month after successful kidney-pancreas transplantation. She had a previous septic thromboembolic occlusion of the kidney allograft artery and underwent an emergency operation under septic conditions. After initial recovery from mitral valve repair, transplant nephrectomy was performed on day 7 after cardiac surgery as a result of ongoing sepsis. She died on day 42 as a result of mucormycosis that was affecting the brain, lungs, and myocardium. At autopsy, there was no recurrence of endocarditis in either transplant patient.

Two male patients in the mitral valve repair group died of unrestrained sepsis. In the replacement group one male patient died of a mesenteric infarction, and 2 patients died of uncontrollable sepsis. Another male lung transplant recipient had an early recurrence of endocarditis and died on the fifth day after reoperation of ongoing sepsis.

\section{Postoperative Outcome}

In the repair group one recurrence of endocarditis occurred 13 months after cardiac surgery. Initially, the patient had destruction of the posterior leaflet and underwent repair with autologous glutaraldehyde-fixed pericardium along 
TABLE 2. Perioperative characteristics

\begin{tabular}{|c|c|c|c|}
\hline & $\begin{array}{l}\text { Mitral valve repair } \\
\quad(\mathrm{n}=34)\end{array}$ & $\begin{array}{l}\text { Mitral valve replacement } \\
\qquad(\mathrm{n}=34)\end{array}$ & $P$ value \\
\hline \multicolumn{4}{|l|}{ Main indication for surgical intervention } \\
\hline Persistent sepsis & $17(50 \%)$ & $6(26.1 \%)$ & .005 \\
\hline Proceeded or imminent septic embolism & $10(29.4 \%)$ & $15(44.1 \%)$ & .2 \\
\hline Congestive heart failure & $7(20.6 \%)$ & $13(38.2 \%)$ & .11 \\
\hline Mean mitral regurgitation (grade SD) & $2.8 \pm 1.2^{*}$ & $2.7 \pm 1.0$ & .957 \\
\hline Positive intraoperative valve culture & $15(44.1 \%)$ & $19(55.9 \%)$ & .332 \\
\hline Staphylococcal endocarditis & $19(55.9 \%)$ & $13(38.2 \%)$ & .145 \\
\hline Leaflet destruction & $15(44.1 \%)$ & $11(32.4 \%)$ & .394 \\
\hline Anterior mitral leaflet & $4(11.8 \%)$ & $5(14.7 \%)$ & \\
\hline Posterior mitral leaflet & $9(26.5 \%)$ & $5(14.7 \%)$ & \\
\hline Both leaflets & $2(5.8 \%)$ & $1(2.9 \%)$ & \\
\hline Perivalvular destruction & $6(17.6 \%)$ & $5(14.7 \%)$ & .701 \\
\hline Chordal rupture & $12(35.3 \%)$ & $8(23.5 \%)$ & .251 \\
\hline Commissure affected & $3(8.8 \%)$ & $4(11.7 \%)$ & .730 \\
\hline \multicolumn{4}{|l|}{ Intraoperative characteristics } \\
\hline Additional surgical procedures & $13(38.2 \%)$ & $13(38.2 \%)$ & 1.0 \\
\hline Coronary artery bypass grafting (procedures) & $6(17.6 \%)$ & $3(17.6 \%)$ & 1.0 \\
\hline Aortic valve replacement (procedures) & $6(17.6 \%)$ & $6(17.6 \%)$ & 1.0 \\
\hline Tricuspid valve repair (procedures) & $3(8.8 \%)$ & $1(2.9 \%)$ & .760 \\
\hline Leaflet reconstruction with direct suture (procedures) & $5(14.7 \%)$ & & \\
\hline $\begin{array}{l}\text { Leaflet reconstruction with glutaraldehyde-fixed pericardium patch } \\
\text { (procedures) }\end{array}$ & $15(44.1 \%)$ & & \\
\hline Annular reconstruction with glutaraldehyde-fixed pericardium patch & $4(11.8 \%)$ & $3(8.8 \%)$ & 659 \\
\hline Mitral ring annuloplasty & $19(55.9 \%)$ & & \\
\hline Chordal implantation-transposition & $5(14.7 \%)$ & & \\
\hline Rectangular resection & $8(23.5 \%)$ & & \\
\hline Sliding leaflet plasty & $4(11.8 \%)$ & & \\
\hline Rotation paracommissural sliding plasty & $3(8.8 \%)$ & & \\
\hline Mechanical mitral valve prosthesis & & $28(82.4 \%)$ & \\
\hline Biologic stented mitral valve prosthesis & & $6(17.6 \%)$ & \\
\hline CPB time (min) & $166.0 \pm 63.2$ & $144.3 \pm 91.0$ & .260 \\
\hline Aortic crossclamp time (min) & $114.3 \pm 52.3$ & $85.5 \pm 40.5$ & .014 \\
\hline
\end{tabular}

$C P B$, Cardiopulmonary bypass. *Mean $\pm \mathrm{SD}$.

with ring annuloplasty. He underwent reoperation under septic conditions after he had an embolic cerebral event and received a mechanical mitral prosthesis. The patient died on the first postoperative day of refractory sepsis. There were no other valve-related complications or late deaths in the repair group. In the replacement group 3 patients underwent reoperation because of recurrence of endocarditis $(2,12$, and 19 months after the primary operation, respectively), and 2 patients died of recurrence of infection 2 and 4 months after initial surgical treatment without undergoing reoperation. Diagnosis of recurrence was confirmed at autopsy. A total of 6 cardiac-related deaths occurred in the replacement group. Three patients died within 18 months, and 3 patients died later in the follow-up.

A male patient underwent reoperation because of thrombosis of the mechanical mitral valve prosthesis and again recovered well. Another patient died of recurrence of sepsis but without reinfection of the valve prosthesis 3 months after the operation.

Actuarial 1-year survival was $88.2 \%$ in the repair group and $73.5 \%$ in the replacement group, and 5-year survival was $85.1 \%$ in the repair group and $66.6 \%$ in the replacement group $(P=.242)$.

One-year freedom from valvular reoperation was $100 \%$ in the repair group and $89.4 \%$ in the replacement group, and at 5 years' follow-up, it was $96.4 \%$ in the repair group and $85.5 \%$ in the replacement group $(P=.146)$.

Actuarial freedom from recurrence of endocarditis at 5 years' follow-up was $96.4 \%$ in the repair group and $82.7 \%$ in the replacement group $(P=.086)$.

\section{Event-Free Survival}

To evaluate the efficacy of both treatment groups, a composite end point consisting of survival, reoperation, and/or 
recurrence of endocarditis was defined to investigate freedom from treatment failure.

Actuarial event-free survival at 1 year was $88.2 \%$ in the repair group and $67.7 \%$ in the replacement group, 5-year event-free survival was $80.4 \%$ in the repair group and $54.6 \%$ in the replacement group, and 10-year event-free survival was $80.4 \%$ in the repair group and $46.2 \%$ in the replacement group $(P=.015$, Figure 1$)$.

\section{Prognostic Factors of Event-Free Survival}

Several clinical parameters referring to preoperative comorbidity and endocarditis-related findings were univariately tested for their effect on event-free survival.

Demographic factors, such as age $(P=.640)$ or sex $(P=$ $.540)$, did not have an influence on event-free survival nor did valve-related parameters, such as staphylococcal infection $(P=.220)$, sepsis as an indication for surgical intervention $(P=.56)$, positive valve cultures at surgical intervention $(P=.404)$, or preoperative septic embolism $(P=$ $.912)$, or renal dysfunction at surgical intervention $(P=$ $.120)$.

However, impaired left ventricular function (ejection fraction $<48 \%$ ) at surgical intervention was associated with poor event-free survival $(P=.002)$, as well as additional surgical procedures, such as coronary artery bypass grafting or other valve procedures $(P=.035)$.

Adjusting for these variables in the multivariate analysis, mitral valve repair remained the superior therapy regarding event-free survival compared with valve replacement (HR, $0.33 ; 95 \%$ CI, $0.12-0.93 ; P=.02)$. In addition, impaired left ventricular function at surgical intervention was independently affecting the event-free survival (HR, 3.0; 95\% CI, 1.099-8.42; $P=.046$ ). However, contrary to univariate analysis, the need for additional surgical procedures did not predict a difference in event-free survival by means of multivariate analysis (HR, 2.1; 95\% CI, 0.88-5.2; $P=.092$ ).

\section{Discussion}

Several outcome studies have confirmed the feasibility of mitral valve repair in IE but were not able to demonstrate the superiority of repair in a severity-matched cohort. ${ }^{6,9,11,14,15}$ Additionally, this is the largest consecutive series of patients described undergoing mitral valve repair in active infection.

To our knowledge, this is the first study demonstrating a significantly improved event-free survival of mitral valve repair over replacement in comparable groups of patients with severe native mitral endocarditis. Even though we found a similar extent of valvular destruction and paravalvular lesions and a significantly higher incidence of septic embolism in the repair group, valve repair was an independent predictor of event-free survival.

In contrast to other studies, ${ }^{7,9-11}$ we only included patients with active mitral valve endocarditis and no patients

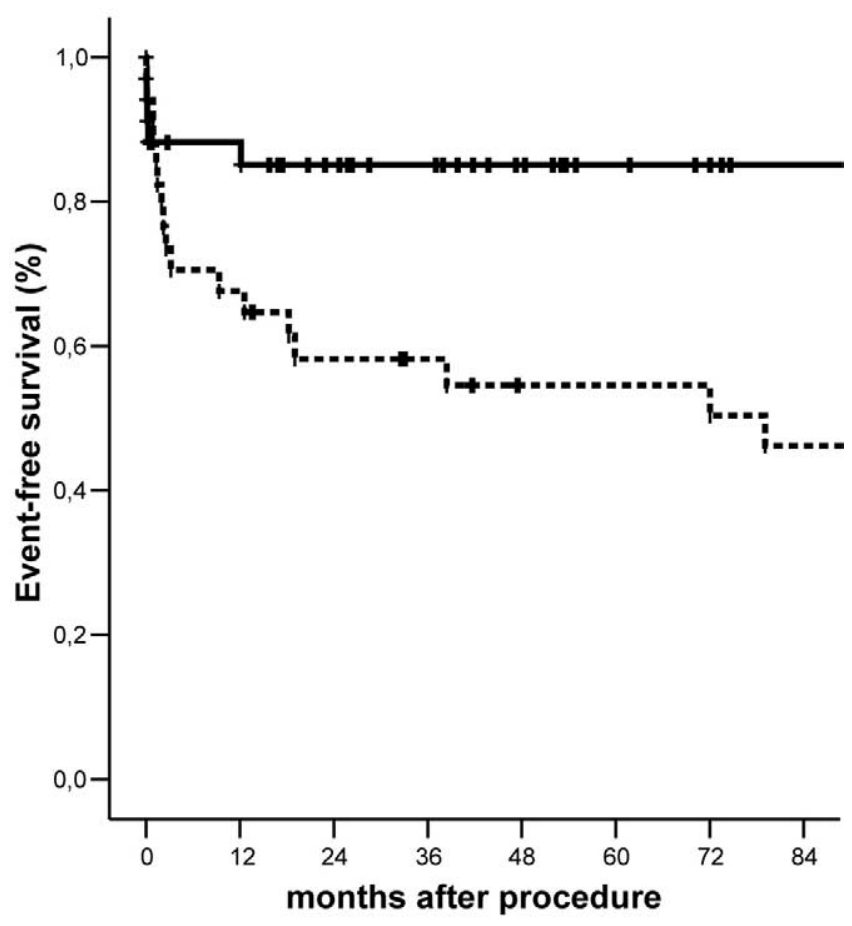

Figure 1. Kaplan-Meier event-free survival (freedom from death, valvular reoperation, and/or recurrence of endocarditis) for patients undergoing either mitral repair (solid line) or replacement (dashed line) for acute mitral endocarditis ( $P=.015$, log-rank test).

with healed endocarditis. Additionally, patients presenting with underlying rheumatic valve disease who are more likely to undergo replacement were not included into this study.

Muehrcke and associates ${ }^{10}$ reported a significantly better event-free survival in the repair group, consisting of mainly healed endocarditis, but we could not confirm this result in the subgroup of 26 patients undergoing mitral repair for acute endocarditis. Additionally, rheumatic valve disease was present in their replacement group.

Although the decision for the surgical strategy is mainly dependent on the extent of valvular and annular destruction, we were able to perform sufficient repair, even in severely destructed mitral valves, including annular abscesses. In contrast to Mihaljevic and coworkers, ${ }^{14}$ who presented a repair group undergoing operation for vegetations only in $81 \%$ of the study group, we were able to perform sufficient repair in patients mainly presenting with severe destruction of at least one mitral leaflet.

The use of prosthetic material in active endocarditis is still controversial. ${ }^{10,16,17}$ However, there has never been a study evidencing that either the choice of prosthesis (mechanical or biologic) or the use of a prosthetic annuloplasty ring is responsible for a higher recurrence rate of endocar- 
ditis. Because underlying valve disease is the main risk factor for the development of IE, optimal repair is essential to prevent recurrence. Therefore we are routinely using prosthetic rings in patients with annular dilatation, even though this annuloplasty might present additional risk for recurrent bacterial colonization. However, we are convinced that a remaining mitral regurgitation bears a higher risk for recurrence than a prosthetic ring annuloplasty.

In our series more than $50 \%$ of all patients received a prosthetic mitral ring annuloplasty, and there was only one recurrence of endocarditis in a male patient with methicillinresistant Staphylococcus aureus 13 months after valve repair. The low recurrence rate in the repair group underlines this strategy; however, radical debridement of all infected and potentially infected material is a precondition.

During the past decade, hospital-acquired endocarditis with highly aggressive micro-organisms has become more frequent in more ill patients (eg, transplant recipients). In our experience the predominant infective organism was Staphylococcus species, and this organism was even more prevalent in the repair group. In agreement with Renzulli and associates, ${ }^{18}$ positive blood and tissue cultures were not predictive of poor event-free survival in our study. However, in contrast to Sternik and associates ${ }^{15}$ patients with staphylococcal endocarditis at the time of the operation were not more likely to undergo valve replacement in our study. In their study the authors demonstrated excellent results, even in patients undergoing complex repairs.

In destructive mitral endocarditis, the concept of prosthetic valve replacement as the established primary treatment option for IE might be substantially wrong, and we have to reassess the therapeutic concepts.

Of course, the surgeon's experience in mitral valve repair is essential, and only highly specialized and skilled surgeons would dare to repair even severely destructed mitral valves. In our patient cohort reconstructive operations were exclusively performed by 2 surgeons highly experienced in mitral repair and might therefore be responsible for the convincing results.

From clinical experience and quality control, we know that rare surgical procedures should be limited to few surgeons to gain experience and excellent results. Because IE is of growing importance in cardiac surgery, the number of specialists in this field should increase. In general, the surgeon's bias might be present in all surgical case series and could be avoided by randomization. However, we believe that forcing a surgeon to perform a procedure of which he or she is not convinced or experienced with at a high level might be unethical.

The restrictive attitude toward surgical intervention in acute endocarditis has changed during the last decade. Early operation for IE is more common today, on the one hand because of improved results of cardiac surgery in general and on the other hand because of the higher incidence of more aggressive forms of endocarditis resulting in septic embolism, perivalvular abscess, and the higher frequency of hospital-acquired endocarditis that is mainly incurable with conservative therapy alone. ${ }^{19}$ Additionally, in acute endocarditis, when the clinical course is complicated by septic cerebral embolism, early surgical intervention is recommended despite the risk of secondary cerebral hemorrhage. Several studies have shown that the cardiac procedure ought to be performed within 72 hours after occurrence of the embolic event. ${ }^{20,21}$ In contrast to the results of Jault and coworkers, ${ }^{22}$ septic embolic complications before cardiac surgery were not associated with a poor event-free survival in our experience.

The nonrandomized design of our study limits our conclusions; however, it is unlikely that randomized trials will be feasible regarding the choice of these 2 surgical techniques. Although the groups seem to be highly comparable, it is very difficult to be sure that the characteristics of the patients and, especially, of the valves are identical. Also, we cannot neglect effects caused by the surgeon's experience. However, the significant differences in late outcome are not related to eventual differences in surgical mortality. Therefore it appears quite evident that the differences found are not due to chance or bias alone.

In conclusion, even though we have to deal with more complex forms of mitral valve endocarditis in more ill patients today, ${ }^{23,24}$ valve repair offers excellent early and late results in patients with active mitral valve endocarditis and is the preferable treatment option in the surgical therapy of native mitral endocarditis.

\section{References}

1. Moreillon P, Que YA. Infective endocarditis. Lancet. 2004;363: 139-49.

2. Hoen B, Alla F, Selton-Suty C, et al. Changing profile of infective endocarditis: results of a 1-year survey in France. JAMA. 2002;288: 75-81.

3. Bouza E, Menasalvas A, Munoz P, Vasallo FJ, del Mar Moreno M, Garcia Fernandez MA. Infective endocarditis: a prospective study at the end of the twentieth century-new disposing conditions, new etiologic agents, and still a high mortality. Medicine (Baltimore). 2001; 80:298-307.

4. Bouza E, Menasalvas A, Munoz P, Vasallo FJ, del Mar Moreno M, Garcia Fernandez MA. Infective endocarditis: a prospective study at the end of the twentieth century-new disposing conditions, new etiologic agents, and still a high mortality. Medicine (Baltimore). 2001; 80:298-307.

5. Dreyfus G, Serraf A, Jebara VA, et al. Valve repair in acute endocarditis. Ann Thorac Surg. 1990;49:706-11.

6. Podesser BK, Roedler S, Hahn R, et al. Mid-term follow up of mitral valve reconstruction due to active infective endocarditis. $J$ Heart Valve Dis. 2000;9:335-40.

7. Pagani FD, Monaghan HL, Deeb GM, Bolling SF. Mitral valve reconstruction for active and healed endocarditis. Circulation. 1996; 94(suppl II):II133-8.

8. Hendren WG, Morris AS, Rosenkranz ER, et al. Mitral valve repair for bacterial endocarditis. J Thorac Cardiovasc Surg. 1992;103:124-9. 
9. Iung B, Rousseau-Paziaud J, Cormier B, et al. Contemporary results of mitral valve repair for infective endocarditis. J Am Coll Cardiol. 2004;43:386-92.

10. Muehrcke DD, Cosgrove DM, Lytle BW, et al. Is there an advantage to repairing infected mitral valves? Ann Thorac Surg. 1997; 63:1718-24.

11. Wilhelm MJ, Tavakoli R, Schneeberger K, et al. Surgical treatment of infective mitral valve endocarditis. J Heart Valve Dis. 2004;13:754-9.

12. Durack DT, Lukes AS, Bright DK. New criteria for diagnosis of infective endocarditis: utilisation of specific echocardiographic findings. Dukes Endocarditis Service. Am J Med. 1994;96:200-9.

13. Harris KM, Li DY, L'Ecuyer P, et al. The prospective role of transesophageal echocardiography in the diagnosis and management of patients with suspected infective endocarditis. Echocardiography. 2003;20:57-62.

14. Mihaljevic T, Paul S, Leacche M, et al. Tailored surgical therapy for acute native mitral valve endocarditis. J Heart Valve Dis. 2004;13: 210-6.

15. Sternik L, Zehr KJ, Orszulak TA, et al. The advantage of repair of mitral valve in acute endocarditis. J Heart Valve Dis. 2002;11:91-8.

16. Aagaard J, Andersen PV. For acute endocarditis treated with radical debridement and implantation of mechanical or stented bioprosthetic devices. Ann Thorac Surg. 2001;71:100-4.
17. Renzulli A, Carozza A, Romano G, et al. Recurrent infective endocarditis: a multivariate analysis of 21 years of experience. Ann Thorac Surg. 2001;72:39-43.

18. Renzulli A, Carozza A, Marra C, et al. Are blood and valve cultures predictive for long-term outcome following surgery for infective endocarditis? Eur J Cardiothorac Surg. 2000;17:228-33.

19. Fernandez-Guerrero ML, Verdejo C, Azofra J, de Gorgolas M. Hospital-acquired infectious endocarditis not associated with cardiac surgery: an emerging problem. Clin Infect Dis. 1995;20:16-23.

20. Hart RG, Foster JW, Luther MF, Kanter MC. Stroke in infective endocarditis. Stroke. 1990;21:695-700.

21. Piper C, Wiemer M, Schulte HD, Horstkotte D. Stroke is not a contraindication for urgent valve replacement in acute infective endocarditis. J Heart Valve Dis. 2001;10:703-11.

22. Jault F, Gandjbakhch I, Rama A, et al. Active native valve endocarditis: determinants of operative death and late mortality. Ann Thorac Surg. 1997;63:1737-41.

23. Lacassin F, Hoen B, Leport C, et al. Procedures associated with infective endocarditis in adults. A case control study. Eur Heart J. 1995;16:1968-74.

24. Horstkotte D, Follath F, Gutschik E, et al. Guidelines on prevention, diagnosis and treatment of infective endocarditis. Executive summary. Eur Heart J. 2004;25:267-76.

\section{Availability of Journal back issues}

As a service to our subscribers, copies of back issues of The Journal of Thoracic and Cardiovascular Surgery for the preceding 5 years are maintained and are available for purchase from Elsevier Inc. until inventory is depleted. Please write to Elsevier Inc., Subscription Customer Service, 6277 Sea Harbor Dr, Orlando, FL 32877, or call $800-654-2452$ or $407-345-4000$ for information on availability of particular issues and prices. 